\title{
Lorentz transformations of open systems
}

\author{
ASHER PERES and DANIEL R. TERNO
}

Department of Physics, Technion-Israel Institute of Technology, 32000 Haifa, Israel

\begin{abstract}
We consider open dynamical systems, subject to external interventions by agents that are not completely described by the theory (classical or quantal). These interventions are localized in regions that are relatively spacelike. Under these circumstances, no relativistic transformation law exists that relates the descriptions of the physical system by observers in relative motion. Still, physical laws are the same in all Lorentz frames.
\end{abstract}

\section{A tale of two magicians}

Many years ago, some time in the twentieth century, there were two itinerant magicians (probably Gypsies) who went from village to village and entertained people with sleight of hand tricks. One of them called himself a quantum magician. He claimed to have supernatural power, to use supersymmetric particles and to send quantum information with superluminal velocity. The crowds were amazed by his supernatural legerdemain.

A few days later, came the second magician. That one wanted to be an educator. He showed how to mimic quantum uncertainties with a mundane deck of cards [1]. He indeed repeated every trick of the quantum magician, and he then explained how to perform it, using only the ordinary laws of classical mechanics. People were dismayed. They realized that they had been fooled: in the preceding shows they had not seen anything supernatural. No one liked the second magician, and no one thanked him for his lessons.

For instance, the quantum magician produced two spin $\frac{1}{2}$ particles in a singlet state, far away from each other. His assistant Alice measured one of the spin components, and instantaneously the wave function collapsed all over spacetime. All over spacetime? This makes no sense. A wave function does not live in spacetime, but in a Hilbert space; and in a Hilbert space, the notion of velocity does not exist. 
The second magician just took a piece of ordinary matter at rest. He made it explode into two fragments which carried opposite angular momenta, whose directions were unpredictable and had an isotropic Liouville distribution. The magician's assistant, called Bob, measured the angular momentum of one of the fragments and, lo and behold, the Liouville distribution instantaneously collapsed all over spacetime. All over spacetime? Of course not. Liouville distributions do not live in spacetime, but in phase space. Phase space has a symplectic structure, and does not admit the notion of velocity.

\section{Two quantum particles}

The twentieth century witnessed two revolutions in our conception of nature. The first one was relativity theory: Einstein found that simultaneity had no absolute meaning and that distant events might have different time orderings when referred to observers in relative motion. Einstein's theory elicited strong opposition when it was proposed, but is generally accepted by now. On the other hand, the revolution caused by quantum theory still produces uneasy feelings among some physicists. Einstein himself was puzzled by what seemed to be instantaneous transmission of quantum information. In his autobiography [2] he used terms such as "telepathically" (p. 85) and "spook" (p. 683).

Since then, the "peaceful coexistence" [3] of special relativity and quantum measurement theory was the subject of numerous theoretical articles [4. The first attempts to discuss the compatibility of both theories were rather naive: the only relativistic feature that was used was the existence of an upper bound, the velocity of light, on the speed of propagation of physical effects. This mere limitation does not do justice to the fundamentally new concepts introduced by Einstein's relativity (one could as well imagine information theory limited by the speed of sound, or that of the postal service).

A more subtle issue, that came under scrutiny in later years, is that a physical situation involving several observers in relative motion cannot be described by wave functions with a relativistic transformation law [5, 6]. This is true even if we allow that law to be nonlocal. (Apparatuses with parts in relative motion have been the subject of recent experiments [7, 8). The root of the difficulty we have to transform quantum expressions

from one Lorentz frame to the other is that the process called "quantum measurement" is an intervention in the quantum dynamics by an "exosystem" [9], namely by an apparatus which is not completely described by the quantum formalism [10].

Consider the following simple example, illustrated by Fig. 1 which shows the world 
lines of two observers ("Alice" and "Bob") who are receding from each other. Two

spin $\frac{1}{2}$ particles are initially prepared in identical states, $\left|\sigma_{x}\right\rangle=1$, far away from each other, in the frame of reference of the quantum magician, where they are at rest. Note that these particles are not entangled: the problem we are discussing has no relation whatsoever to quantum entanglement. Alice and Bob, as seen in that frame, move in opposite $\pm x$ directions. They simultaneously measure the values of $\sigma_{y}$ of their particles, in regions $\mathrm{A}$ and $\mathrm{B}$ respectively. Actually these are not quite the same variables $\sigma_{y}$, because Alice and Bob are in relative motion in the $x$ direction and there is a transformation law between their operators $\sigma_{y}$ [11]. However, this detail is irrelevant in the present discussion. The main point is that their results, \pm 1 , are equiprobable and unpredictable, except statistically.

For example, suppose that Alice finds $\sigma_{y A}=1$ and Bob finds $\sigma_{y B}=-1$. Then at time $t_{A}=0$ (in Alice's frame), namely after Alice performed her measurement but before Bob performed his, the state of the physical system is described by her as

$$
\left|\psi\left(t_{A}=0\right)\right\rangle=\left|\left(\sigma_{y A}=1\right)\right\rangle \otimes\left|\left(\sigma_{x B}=1\right)\right\rangle .
$$

(Alice knows that $\sigma_{x B}=1$ because she is cognizant of the preparation procedure of the two particles.) Likewise, the state at time $t_{B}=0$ is described by Bob as

$$
\left|\psi\left(t_{B}=0\right)\right\rangle=\left|\left(\sigma_{x A}=1\right)\right\rangle \otimes\left|\left(\sigma_{y B}=-1\right)\right\rangle .
$$

No relativistic linear transformation can convert these expressions into one another, in a way that would be valid for all possible results $\sigma_{y}= \pm 1$.

\section{Two classical particles}

We shall now show that a similar situation arises for a classical system whose state is given in any Lorentz frame by a Liouville function [12]. Recall that a Liouville function expresses our probabilistic description of a physical system - what we can predict before we perform an actual observation - just as the quantum wave function is a mathematical expression used for computing probabilities of events 13.

To avoid any misunderstanding, we emphasize that there is no consistent relativistic statistical mechanics for $N$ interacting particles, with a $6 N$-dimensional phase space defined by the canonical coordinates $\mathbf{p}_{n}$ and $\mathbf{q}_{n}(n=1, \ldots, N)$. Any relativistic interaction must be mediated by fields, having an infinity of degrees of freedom. (The same is true in 
quantum mechanics: we need quantum field theory to have interactions compatible with special relativity.) A complete Liouville function, or rather Liouville functional, must therefore contain not only all the canonical variables $\mathbf{p}_{n}$ and $\mathbf{q}_{n}$, but also all the fields. However, once this Liouville functional is known (in principle), we can define from it a reduced Liouville function, by integrating the functional over all the degrees of freedom of the fields. The result is a function of $\mathbf{p}_{n}$ and $\mathbf{q}_{n}$ only (just as we have reduced density matrices in quantum theory). The time evolution of such reduced Liouville functions cannot be obtained directly from canonical Hamiltonian dynamics, without explicitly mentioning the fields, yet these functions are well defined in any Lorentz frame, and their relativistic transformation is unambiguous [12].

As a simple example, consider two equal point masses $m$, identically prepared by the second magician, far away from each other. These masses are restricted to move along straight segments with fixed positions on the $x$-axis in the magician's frame. In that frame, the masses move with constant velocity and they bounce elastically when they reach the extremities of their segments. Their momenta thus are $\pm p$, and their energy is $E_{0}=\left(m^{2}+p^{2}\right)^{1 / 2}$. At some arbitrary time $t$ in the magician's frame, each mass receives a kick $k$, due to an external agent, so that its momentum becomes $k \pm p$. Its energy becomes

$$
E_{ \pm}=\sqrt{m^{2}+(k \pm p)^{2}}
$$

As before, Alice and Bob have opposite velocities with respect to the above inertial frame, and they wish to describe the dynamical evolution of the physical system in terms of their times, $t_{A}$ and $t_{B}$, respectively. The problem is that they know only statistically the sign of $\pm p$ before the kick, and therefore the correct sign to use in Eq. (3).

Let us assume that the kicks $k$ occurred in spacetime regions A and B like those in Fig. 1. Then, according to Alice, the situation at time $t_{A}=0$ is the following: the particle that was kicked in region $A$ has equal probabilities to have energy $E_{1}=E_{ \pm}$, and the other particle still has energy $E_{0}$ with certainty. (These are the values of the energies in the magician's frame. They may be transformed to Alice's frame if we wish to do so.) The Liouville function, averaged over all the variables except the energies of the two particles, is concentrated in two points as shown in Fig. 2(a). If Alice actually measures $E_{1}$, one of the two points disappears. Bob's description, on the other hand, is given by Fig. 2(b). Likewise, if he measures $E_{2}$, one of the two points of Fig. 2(b) disappears. No Lorentz transformation of the Liouville function [12] can relate these different descriptions. 
Note that for spacelike planes that intersect the past light-cones of events $A$ and $B$, the Liouville function is concentrated at a single point in the $E_{1} E_{2}$ plane, namely $E_{1}=E_{2}=$ $E_{0}$. For those intersecting the future light-cones of both events, the Liouville function has a support consisting of four points if no measurement is performed, because both $E_{1}$ and $E_{2}$ can have values $E_{ \pm}$. It is only for spacelike planes that intersect the future light cone of one of these regions, and the past light cone of the other one, that we get two incompatible descriptions, if measurements are performed at times $t_{A}$ and $t_{B}$, respectively.

\section{Open systems}

The purpose of this article was to show that similar problems (or "paradoxes") occur in classical and quantum relativistic dynamics, if there are incompletely described physical agents for which we have only probabilistic data. This is a general property of open physical systems, irrespective of the details of their dynamics.

In real life, there are no closed physical systems. We may, if we wish, imagine that closed systems exist, but since there is no communication with them, their properties are irrelevant. Still, when we learn (or teach) physics, physical laws are usually formulated in a language appropriate to closed systems. For example, Maxwell's equations are written

in terms of vectors $\mathbf{E}$ and $\mathbf{B}$. The components of these vectors have no objective physical meaning. Their values depends on the choice of the coordinate system. On the other hand, it is impossible to formulate the dynamical laws in terms of the scalar quantities $\mathbf{E}^{2}-\mathbf{B}^{\mathbf{2}}$ and $\mathbf{E} \cdot \mathbf{B}$. We need $\mathbf{E}$ and $\mathbf{B}$ explicitly. Therefore, a complete description would have to include not only the components of the electromagnetic field, but also the material realization of the coordinate system used for defining these vector components. When we say that Maxwell's equations are rotationally invariant, this actually means that if the material rods that serve us as coordinate axes are rigidly rotated, then the components of $\mathbf{E}$ and $\mathbf{B}$ have to be replaced by appropriate linear combinations, in such a way that the equations have the same appearance, in terms of the new coordinates and new field components.

Moreover, to give a physical meaning to the symbols $\mathbf{E}$ and $\mathbf{B}$, we should specify how their numerical values are actually measured, say by means of electrometers and magnetometers, or other suitable instruments. Now, normally we don't want to be bothered by how the field components are actually measured, even less by how the spacetime coordinates are materialized. These technical details are, after all, irrelevant to electromagnetic 
theory. It is tacitly assumed that coordinates are well defined and that precise measuring instruments exist at every spacetime point, and we can proceed with the calculations without having to think about them. However, as shown in this paper, what is acceptable and convenient in a deterministic classical theory becomes problematic if stochastic features are present in the dynamical evolution, as it happens in quantum phenomena and also in classical statistical mechanics. The "spooky" long range quantum correlations that were mentioned by Einstein [2] also appear in classical systems if the latter are incompletely specified and treated statistically by means of Liouville functions (correlations have a meaning only in statistical analyses).

The crucial feature common to both models discussed above is the intervention of "exosystems" [9] (the measuring apparatus and the $k$-kicker) that are not described by the dynamical formalism of the "endosystem" under consideration, and induce a stochastic behavior of the latter. The point is that a physical system is "open" when parts of the universe are excluded from its description. At time $t_{A}=0$, one of the exosystems has intervened at $\mathrm{A}$ but the other one, that did not intervene as yet, is irrelevant and need not be excluded. Conversely, at time $t_{B}=0$ it is the first exosystem that is irrelevant and need not be excluded. Therefore at times $t_{A}=0$ and $t_{B}=0$ different parts of the universe are excluded, the two systems are different, and this explains why no Lorentz transformation exists that relates them. This argument can also be rephrased in terms of quantum contextuality [14].

It is noteworthy that another causality problem is related to stochastic exosystems: causal loops (that is, hypothetical effects of future events on past ones) are dynamically inconsistent if, and only if, we allow exophysical agents to influence the evolution of physical systems [15, 16]. For closed systems, fully described by the theory, causal loops lead to no inconsistency and there is effectively no difference between past and future.

\section{Concluding remarks}

Just as the second magician, we don't expect anyone to thank us for dispelling some of the "paradoxes" of the quantum folklore. It is unfortunate that these fallacious paradoxes are often invoked as a source of dissatisfaction with the axioms of quantum mechanics. We have shown that similar features also appear in classical theory. They are solely due to the introduction of exosystems, which are necessary for the interpretation of the theory, but are not described by the latter [17. 
One may be tempted to say that it is stochasticity, not quantum theory, that requires "peaceful coexistence" [3] with special relativity. However, there is more in quantum theory than just the existence of probabilistic data. A true quantum magician, using Bell's theorem [18], would be able to display long range correlations that cannot be reproduced by a classical magician. Bell's theorem implies severe restrictions on our ability to have together realism and locality. The essential point we wanted to make in this paper is that in discussions of these fundamental issues, the fact that physical systems are open should never be forgotten.

\section{Acknowledgments}

We thank Larry Schulman and Abner Shimony for helpful comments. Work by AP was supported by the Gerard Swope Fund and the Fund for Encouragement of Research. DRT was supported by a grant from the Technion Graduate School.

\section{References}

[1] Kirkpatrick, K. A., 2001, e-print quant-ph/0106072.

[2] Schilpp, P. A., ed., 1949, Albert Einstein: Philosopher-Scientist (Evanston: Library of Living Philosophers).

[3] Shimony, A., 1978, Internat. Philos. Quarterly, 18, 3.

[4] Wheeler, J. A. and Zurek, W. H., eds., 1983, Quantum Theory and Measurement (Princeton: Princeton University Press).

[5] Peres, A., 1995, Quantum Theory: Concepts and Methods (Dordrecht: Kluwer), p. 154.

[6] Peres, A., 1995, in Fundamental Problems in Quantum Theory, ed. by D. M. Greenberger and A. Zeilinger, Ann. New York Acad. Sci., 755, 445; and references therein.

[7] Scarani, V., Tittel, W., Zbinden, H., and Gisin, N., 2000, Phys. Lett., A, $276,1$.

[8] Zbinden, H., Brendel, J., Gisin, N., and Tittel, W., 2001, Phys. Rev., A, 63, 022111. 
[9] Finkelstein, D., 1988, in The Universal Turing Machine. A Half-Century Survey, ed. by R. Herken (Oxford: Oxford University Press), pp. 349-376.

[10] Peres, A., 2000, Phys. Rev., A, 61, 022116; ibid. 61, 022117.

[11] Thomas, L. H., 1927, Phil. Mag., 3, 1.

[12] Balescu R. and Kotera, T., 1967, Physica, 33, 558.

[13] Some physicists hold the opinion that wave functions are physical entities, not only mathematical tools for computing probabilities.

[14] Khrennikov, A., 2001, e-print quant-ph/0106073.

[15] Peres, A., and Schulman, L. S., 1972, Int. J. Theor. Phys., 6, 377.

[16] Gatuin, L. L., 1980, Int. J. Theor. Phys., 19, 25.

[17] Peres, A. and Zurek, W. H., 1982, Am. J. Phys., 50, 807.

[18] Bell, J. S., 1964, Physics, 1, 195.

FIG. 1. Events that occur simultaneously (in the magicians' frame) in regions A and $B$ have different time orderings when described in the Lorentz frames of Alice and Bob.

FIG. 2. Liouville functions, projected on the energy plane $E_{1} E_{2}$, at times $t_{A}=0$ and $t_{B}=0$, respectively, if no measurements are performed to resolve the indeterminacy. The dots indicate values of $E_{1}$ and $E_{2}$ for which the value of the Liouville function does not vanish. 

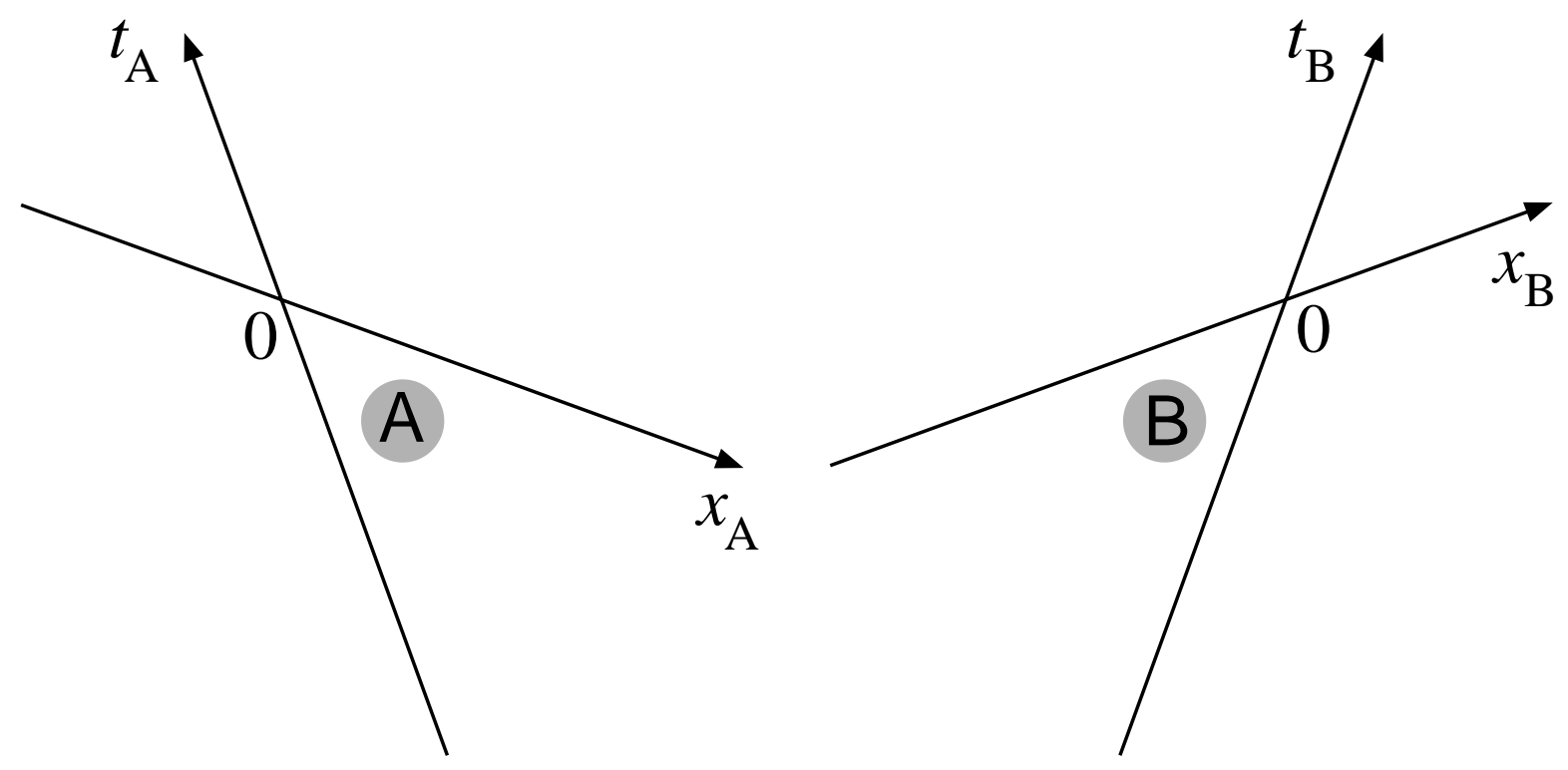

Figure 1
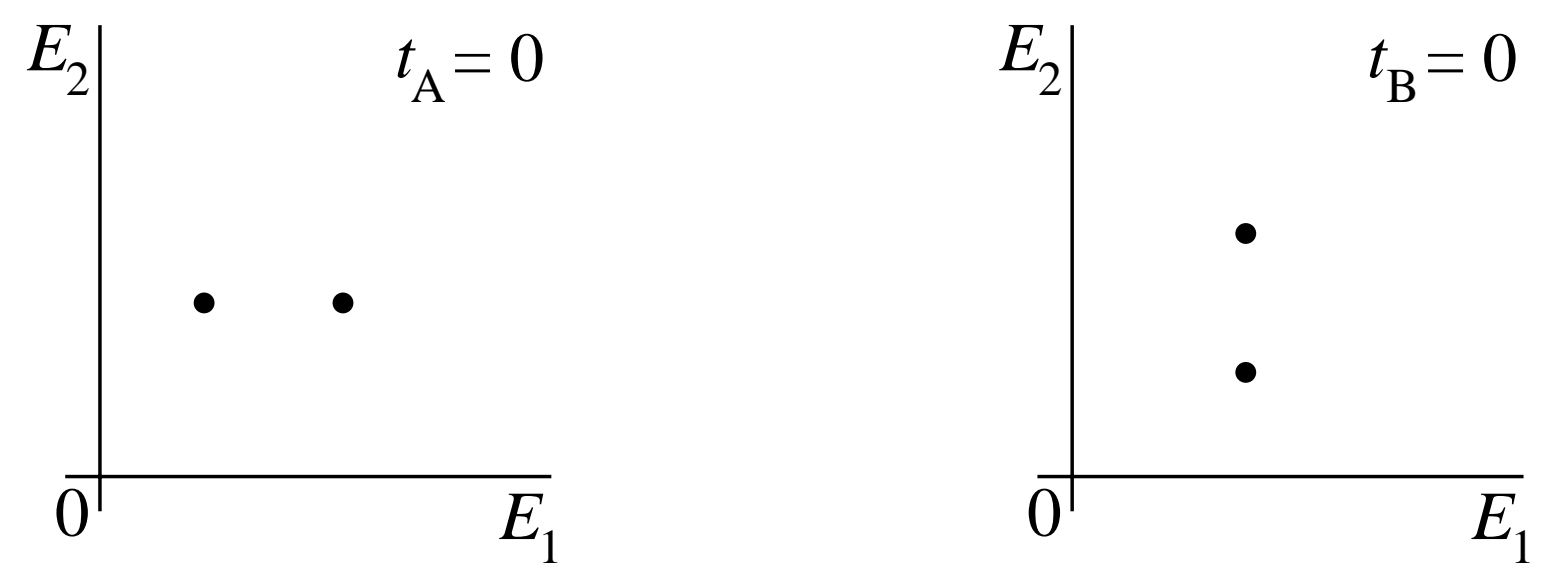

Figure 2

Peres and Terno - Lorentz transformations of open systems 